\title{
Results of neutrino point source searches with 2008-2014 IceCube data above $10 \mathrm{TeV}$
}

\author{
The IceCube Collaboration \\ ${ }^{\dagger}$ http://icecube.wisc.edu/collaboration/authors/icrc15_icecube
}

E-mail: coendersaicecube.wisc.edu

\begin{abstract}
The emphasis on point-like source searches for astrophysical neutrinos has recently been strengthened by the unambiguous detection of high-energy astrophysical neutrinos by IceCube. So far, the limited statistics and angular resolution of the relevant analyses do not resolve more than an isotropic emission of astrophysical neutrinos. We present the results of searches for neutrino emission of point-like sources using six years of integrated IceCube livetime, of which three years use the complete IceCube detector. Focusing on track-like events induced by chargedcurrent muon-neutrinos, a large statistics sample of $\sim 600000$ events on the full sky with median angular resolution between $1^{\circ}$ and $0.4^{\circ}$, improving with higher energy, has been collected. For the southern hemisphere, the main background consists of bundles of muons created in extensive air-showers in the Earth's atmosphere, whereas the northern hemisphere is dominated by neutrinos created in the same process. Using an unbinned likelihood maximization search for local clustering, IceCube is sensitive to sources in the northern sky with fluxes substantially below $E^{2} \partial \phi / \partial E=10^{-12} \mathrm{TeV} \mathrm{cm}^{-2} \mathrm{~s}^{-1}$. We report about the status of these searches and the implications on the nature of the observed flux as well as on single source candidates.
\end{abstract}

Corresponding authors: Stefan Coenders ${ }^{* 1}$, Elisa Resconi ${ }^{1}$

${ }^{1}$ Technische Universität München, Physik-Department, Boltzmannstr. 2, 85748 Garching

The 34th International Cosmic Ray Conference,

30 July- 6 August, 2015

The Hague, The Netherlands

${ }^{*}$ Speaker. 


\section{Introduction}

IceCube is a cubic-kilometer neutrino detector installed in the ice at the geographic South Pole [1] between depths of $1450 \mathrm{~m}$ and $2450 \mathrm{~m}$. Detector construction started in 2005 and finished in 2010. Neutrino reconstruction relies on the optical detection of Cherenkov radiation emitted by secondary particles produced in neutrino interactions in the surrounding ice or the nearby bedrock. During construction, partial detector configurations were operational taking data with 40 strings from April 2008, 59 strings from May 2009, and 79 strings from May 2010 on before IceCube was fully operational in May 2011.

The observation of high-energy astrophysical neutrinos by IceCube in events starting in the detector volume $[2,3]$ as well as up-going muons created in charged-current muon-neutrino interactions outside the detector $[4,5]$ strengthens the importance of searches for point-like sources of neutrinos of astrophysical origin. Neutrinos are the ideal astrophysical messenger to study acceleration mechanisms of Cosmic Rays (CRs). In hadronic interactions, neutrinos are produced alongside photons and charged particles, but due to their neutral charge and low interaction crosssection, neutrinos are neither deflected by magnetic fields, nor absorbed by interstellar media. If hadronic processes are part of the acceleration mechanisms of cosmic rays, both gamma-rays and neutrinos are produced in the decay of neutral or charged pions, respectively. Hence, using observed gamma-ray observations, predictions about the neutrino emission can be made. Almost independent of the emission spectrum of neutrinos at the source, neutrino oscillations will convert a significant amount (approximately 1/3) of the flux into $v_{\mu}+\bar{v}_{\mu}$ events that produce track-like signatures in IceCube. The first detection of a cosmic neutrino point-like source will represent a major breakthrough and will provide unique insides into aspects of cosmic ray sources.

Presently, the astrophysical signal is compatible with an isotropic distribution, which might be because of the low statistics and large angular uncertainties in the case of events starting in the detector [2], or vast atmospheric backgrounds for through-going muon searches [4, 5, 6, 7]. In order to study in more detail this new neutrino signal, dedicated point-like source searches are performed. Muons induced by high energy $v_{\mu}$ penetrate through several kilometers in the Antarctic ice and hence IceCube collects muons from an effective volume much larger than the instrumented volume. Although the energy information on these through-going events is only a lower bound on the original neutrino energy, their high statistics and excellent angular uncertainty render them ideal candidates for the search for point-like sources of neutrino emission.

The main background for the search of steady neutrino sources comes from neutrinos and muons produced in the atmosphere by extensive air showers. Integrating over long enough time, a steady neutrino source will reveal itself by an increasing excess of events around the source direction over an isotropic background. Furthermore, source models and the observed astrophysical neutrino signal predict a hard energy spectrum for neutrino production at the source $\left(\sim E^{-2}\right)$, whereas the atmospheric background follows a much softer spectrum $\left(\sim E^{-3.7}\right)$.

We present here results from an unbiased all-sky scan and the search for neutrino emission from a limited list of known non-thermal objects. The analyses presented are based on a sample integrated over a livetime of 2063 days. This yields an event sample of approximately 600000 events, providing the currently best neutrino point-like source sensitivity at energies above $100 \mathrm{TeV}$. 

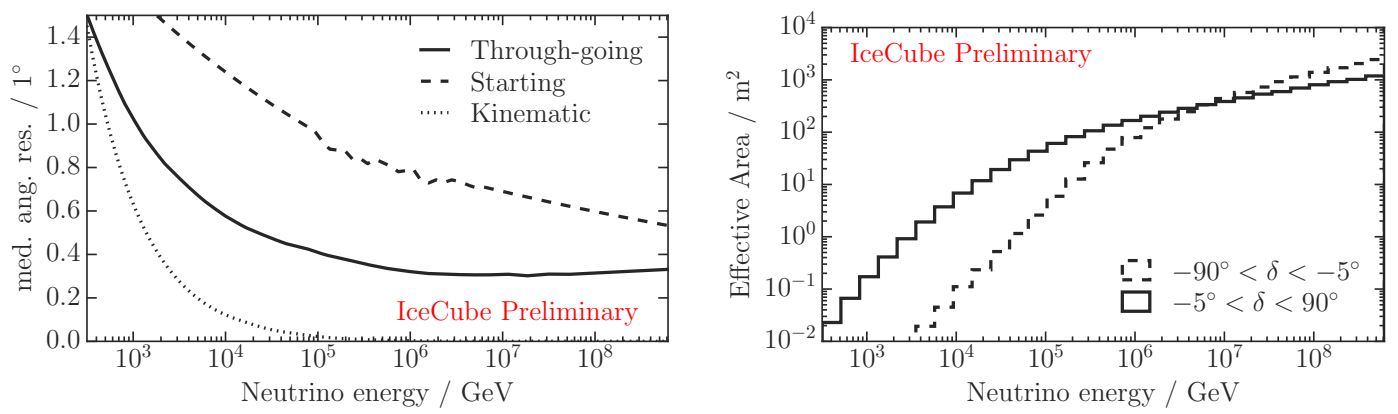

Figure 1: Angular uncertainty (left) and effective area (right) of the IceCube through-going muon point-like source analysis. The left plot also shows the intrinsic directional uncertainty due to the kinematic angle between the muon and primary neutrino. Reconstruction resolution is shown for two event topologies, namely through-going muons and muons that start within the detector. The effective area is shown for up(solid) and down-going (dashed) events separately.

\section{Detector Performance}

The searches for point-like neutrino sources presented here are performed on a sample of through-going muons. At high energies, the muon direction is well correlated with the primary muon-neutrino direction, and the muon itself is reconstructed with an angular uncertainty well below $1^{\circ}$. The median angular resolution is shown in Fig. 1, displaying both the kinematic limit of the neutrino interaction and the reconstruction accuracy of the muon direction with respect to the neutrino direction versus the reconstructed neutrino energy. Increasing with energy, more light is deposited in the detector, giving more information about the event and thus allowing for a better reconstruction.

The event selection techniques applied here are described in $[6,7]$ in detail. After triggering, the over-all background rate is reduced by applying cuts on the quality of the track reconstruction against misreconstructed events. In the atmospheric muon dominated southern hemisphere (downgoing), an additional charge threshold of 1000 photoelectrons (p.e.) is applied to reduce the event rate to the level of the other hemisphere. Subsequently, multivariate selection criteria are applied in order to select well reconstructed muons which carry the most relevant information for point-like source searches; the selection is split for the two hemispheres, taking into account the different backgrounds present there. The selection for each hemisphere is discussed in the following paragraphs.

In the northern hemisphere and the region up to $5^{\circ}$ above the horizon (up-going neutrinos), a pure sample of muon-neutrino induced track-like events is selected using Boosted Decision Tree (BDT) techniques. The surrounding ice and Earth matter shield IceCube from atmospheric muon events; up-going muons can only be a result of a prior charged-current neutrino interaction. Nevertheless, the main background consists of muons that enter from above but are falsely reconstructed as up-going in the detector. A second background for the search of astrophysical sources consists of atmospheric neutrinos that are created in decays of charged $\pi^{ \pm}$and $K^{ \pm}$. Atmospheric neutrinos form an irreducible component of the background and can only be disentangled statistically from the signal of high-energy astrophysical neutrinos, because atmospheric events follow a softer 
spectrum than the expected signal. The background of misreconstructed muons can effectively be removed by BDTs using reconstruction quality parameters. The multivariate classification is trained using experimental data as background, which is dominated by misreconstructed muons, and Monte Carlo simulation of muon neutrinos that interact via charged-current and are reconstructed to within $3^{\circ}$ with respect to the primary neutrino. Two BDTs are trained using different power-law energy spectra $E^{-2}$ and $E^{-2.7}$ benchmarking extra-galactic and galactic scenarios, respectively. Using eleven variables connected to track-quality, event topology, and event-quality, effectively all muons can be rejected as misreconstructed. The final cut on the BDT score is optimized to give the best discovery potential for both hard extra- $\left(E^{-2}\right)$ and softer galactic $\left(E^{-2.7}\right)$ energy spectra.

In the southern hemisphere, the picture is different, which is why an independent selection is performed: Following the primary cosmic-ray energy spectrum, the muon rate falls more rapidly with energy than the expected high energy astrophysical neutrino flux. Consequently, the signal to background rate improves with energy. However, cosmic ray induced air showers produce bundles of muons with high multiplicity. In the detector, this deposits large amounts of energy, faking a single high-energy muon signature. Nevertheless, in contrast to single muons, a bundle produces light by superposition of many Cherenkov-cones washing out the photon arrival times at IceCube's optical sensors (Digital Optical Modules, DOMs). Besides, the energy deposition is smooth over the track distance, whereas a single high-energy muon looses energy via radiative losses showing a large stochasticity along the track. Utilizing this information of photon arrival times with respect to that of a single muon and the differential energy depositions along the track, IceCube has some power to distinguish the signal of single muons at high energies from bundles of atmospheric muons. In the BDT training procedure this is utilized using four different variables, two for the arrival times, and two for the energy depositions. Eight additional variables are chosen to select events that show the best reconstruction and event quality for point-like source related searches. Due to the vast background, the energy-threshold in the down-going region is very high, and only one BDT is trained for hard spectra $\left(E^{-2}\right)$.

In addition to through-going muon events at very high energies, events starting in IceCube can be used to provide additional sensitivity towards point-like sources at lower energies. This is achieved using an active veto of the outer layer of DOMs as described in [2]. Most of the atmospheric muons can be identified as entering IceCube from outside and are thereby rejected, as well as atmospheric neutrinos that are accompanied by muons created in the development of the same shower [8]. In contrast to diffuse searches [2,3], point-like source searches can allow more background because the expected signal is clustering on small scales (below $1^{\circ}$ ), whereas the background is distributed over the entire sky. Moreover, a large off-source region can be used to constrain the background using a data-driven approach. Thus, using a charge threshold of 1500 p.e. for track-like events, 549 starting events are observed within 988 days. These events have a higher probability to be of astrophysical origin than through-going events because the background rate is significantly reduced by using the veto. However, due to a smaller lever arm, the uncertainty of the reconstructed direction is worse than for through-going events as shown in Fig. 1.

Using the selection described above, the final sample consists of an almost pure muon neutrino sample at $2.4 \mathrm{mHz}$ in the northern hemisphere (up-going). For the southern hemisphere, the final rate is $1.1 \mathrm{mHz}$ which is about half of that in previous years. The difference is due to the starting 


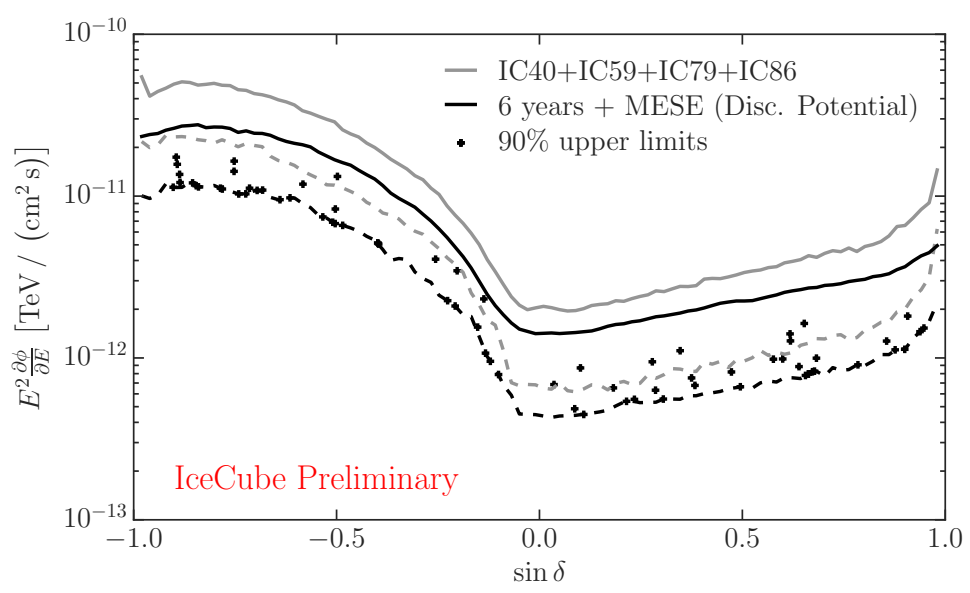

Figure 2: IceCube sensitivity (dashed) and discovery potential (solid) for steady point-like sources using six years of accumulated data. This analysis (black) adds two years to the previous analysis [7] (gray). Black crossed indicate $90 \%$ upper limits for selected source candidates at their declinations.

event sample which is more efficient at lower energies. In total, 600585 events are recorded over the full sky within 2063 days of livetime.

\section{Method}

The sky is probed for local clustering using an unbinned likelihood maximization

$$
L=\prod_{i} \prod_{j \in i}\left(\frac{n_{s}^{j}}{N} S\left(\left|\mathbf{x}_{j}-\mathbf{x}_{S}\right|, E_{j}, \sigma_{j} ; \gamma\right)+\left(1-\frac{n_{s}^{j}}{N}\right) B\left(\delta_{j}, E_{j}\right)\right)
$$

for all events $j\left(\right.$ total $N$ ) at location $\mathbf{x}_{j}$ (declination $\delta_{j}$ ), with energy $E_{j}$ and reconstruction uncertainty $\sigma_{j}$, to be associated with a source at position $\mathbf{x}_{S}$ and spectral index $\gamma$. The signal probability $S$ assumes Gaussian uncertainties in the reconstruction at the source location $\mathbf{x}_{S}$. The background probability is obtained using experimental data from a large fraction of the sky as off-source region. In addition to the event position relatively to the source, energy information of each event is used to discriminate a source with hard power-law $E^{-\gamma}$ from background that is following an atmospheric spectrum. Different detector configurations (partial with respect to full operation) and event selections (starting events versus through-going) are denoted with $i$ and their corresponding expected neutrino rates are taken into account by distributing the total number of clustered events $n_{s}$ among the samples: $n_{s}=\sum_{i} n_{s}^{i}$ assuming a source with power-law spectrum $\propto E^{-\gamma}$.

The likelihood (3.1) is maximized with respect to the null hypothesis $n_{s}=0$ that no significant clustering is observed. The ratio of best fit to null hypothesis forms the test statistic from which the p-value is calculated. For IceCube located at the South Pole, scrambling events in right ascension yields a perfect pure background sample that is used to construct p-values at each point.

Figure 2 shows the sensitivity and discovery potential for six years of IceCube livetime. The sensitivity is defined as the signal flux for which $90 \%$ of experiments yield a test statistics value that is equal or higher than the median value from background fluctuations only, the discovery potential 
is defined as the source flux which yields a p-value at the $5 \sigma$ level in $50 \%$ of the experiments. For the first time, the sensitivity surpasses $10^{-12} \mathrm{TeV} / \mathrm{cm}^{2} \mathrm{~s}$ in most of the northern hemisphere assuming an unbroken $E^{-2}$ spectrum.

\subsection{All Sky Scan}

Using events distributed over the entire sky, the full sky except for a small region $5^{\circ}$ around the poles is scanned for possible clustering. The likelihood (3.1) is maximized for the number of events $n_{s}$ associated to the point that is probed, as well as the spectral index $\gamma$ of the source. The grid used for scanning the sky is chosen to match the typical resolution, using $0.5^{\circ}$ spacing at the equator. The most interesting points (hotspots) are followed up with a finer scan using a width below $0.1^{\circ}$.

For each hemisphere, the most significant spot on the sky is selected according to its p-value using the test-statistic distribution of background scrambled skymaps. Those hotspots are trial corrected by repeating this procedure on the entire sky for pure background samples.

\subsection{A Priori Source list}

In addition to a full sky scan, two source lists of interesting non-thermal candidates were chosen a-priori containing promising candidates for neutrino emission at high energies. The candidates are known by gamma-ray observations and fall into various categories, including galactic sources like pulsar wind nebulae, supernovae remnants, etc., or extra-galactic components as BL Lacs, FSRQs, etc. Both source lists contain less than 50 sources. Consequently, the trial factor is significantly reduced compared to the full sky search, which approximately consists of 100000 to 120000 independent points per hemisphere.

The first source list is similar to the one used in $[6,7]$, setting the focus on the northern hemisphere where IceCube is most-sensitive over a wide-energy range, while only the most promising sources in the southern hemisphere are included as the energy threshold is higher. The source list contains 44 sources, of which 14 are of galactic origin.

The second source list consists of 31 sources in the southern hemisphere, the major part (21) being galactic. This list includes sources that are expected to have softer energy spectra than those in the first list. For these starting events (with their superior background suppression) are better suited than through-going muons. Using the starting event approach based on the veto technique used in [2], the energy threshold can be lowered to $100 \mathrm{TeV}$. With different techniques [9, 10], IceCube can reach lower energies in the southern hemisphere.

For both lists, the most interesting source is trial corrected using background scrambled datasets to obtain the post-trial p-value. This is done for northern and southern sources separately. Hence, three p-values are calculated from the individual source lists.

\section{Results}

In the analysis of six years through-going track data of the IceCube neutrino observatory, no clustering significantly above background expectation was found. Both the most interesting points in the all-sky search as well as the gamma-ray source list are compatible with background expectation. 
Table 1: Selected source candidates of the two a-priori lists. Listed are the post-trial p-value, the fit result of the unbinned likelihood, and an upper limit on an unbroken $E^{-2}$ spectrum $\phi_{v_{\mu}+\bar{v}_{\mu}}^{90 \%}$ in units of $\mathrm{TeV} / \mathrm{cm}^{2} \mathrm{~s}$. Post-trial p-Values are quoted only for the most significant sources per list and hemisphere.

\begin{tabular}{lrlr}
\hline \hline Source & $n_{s}$ & p-Value & $\phi_{v_{\mu}+\bar{v}_{\mu}}^{90{ }^{\prime}}$ \\
\hline Cygnus A & 4.0 & 0.89 & 1.63 \\
PKS 1406-076 & 12.9 & 0.051 & 2.32 \\
PKS 0727-11 & 6.6 & 0.64 & 3.46 \\
Mrk 421 & 5.5 & - & 1.27 \\
Crab Nebula & 6.8 & - & 0.75 \\
\hline \hline
\end{tabular}

In the northern hemisphere, the most significant spot is located at $\alpha=249.6^{\circ}, \delta=63.6^{\circ}$. The best fitting parameters at this point yield $n_{s}=29$ and $\gamma=2.1$ with a pre-trial $\mathrm{p}$-Value of $-\log _{10} \mathrm{p}=$ 5.75. After trial-correction, the post-trial p-Value equals $35 \%$. In the southern hemisphere, the best fitting point of $n_{s}=19, \gamma=2.3$ is located at $\alpha=300.4^{\circ}, \delta=-33.2^{\circ}$. The corresponding pre-trial p-Value of 4.74 results in a trial corrected post-trial p-Value of $87 \%$, that is, well compatible with background expectation.

Regarding the two a priori source lists, all outcomes can be explained with a pure background scenario as well. The most interesting candidate, the FSRQ PKS 1406-076, has a post-trial p-Value of $5.1 \%$. This source is part of the southern part of the first source list used in this analysis. The most interesting sources are listed in Tab. 1.

\section{Sensitivity to Neutrino point-like source Models}

In addition to probing every point in the sky for clustering of neutrinos, neutrinos and gammarays can be correlated by hadronic interactions in the acceleration of cosmic rays. Here, we discuss two promising sources: the Crab nebula as the most powerful TeV gamma-ray source in the sky, and Mrk421, the closest Blazar as extra-galactic source. Model predictions connect the observed gamma-ray spectrum [11] or use Monte Carlo simulation of inelastic proton scattering at the source [12] to make predictions about the neutrino spectrum. IceCube observes small clustering at the position of the Crab nebula (Tab. $1, \delta=22.01^{\circ}, \alpha=83.63^{\circ}$ ), resulting in a $90 \%$ upper limit above the sensitivity. In the case of [11], IceCube's limit is $30 \%$ above the predicted flux, whereas the limits of IceCube constrain the harder flux of [12]. Figure 3 (left) shows the upper limits of IceCube for the predictions of neutrino emission at the position of the Crab nebula.

For Mrk421, the authors of [13] derive a neutrino spectrum based on a fit to the multiwavelength photon spectrum of the source. At the location of Mrk421 $\left(\delta=38.21^{\circ}, \alpha=166.11^{\circ}\right)$ IceCube's upper limit surpasses this model prediction by $16 \%$ (Fig. 3).

\section{Conclusion}

After starting full operation in May 2011, three years of data using the full 86-string detector have been used in the search for steady point sources. This is supplemented by three years of data with the detector in partial configuration, adding up to a total of 2063 days of detector livetime and 600585 events. 

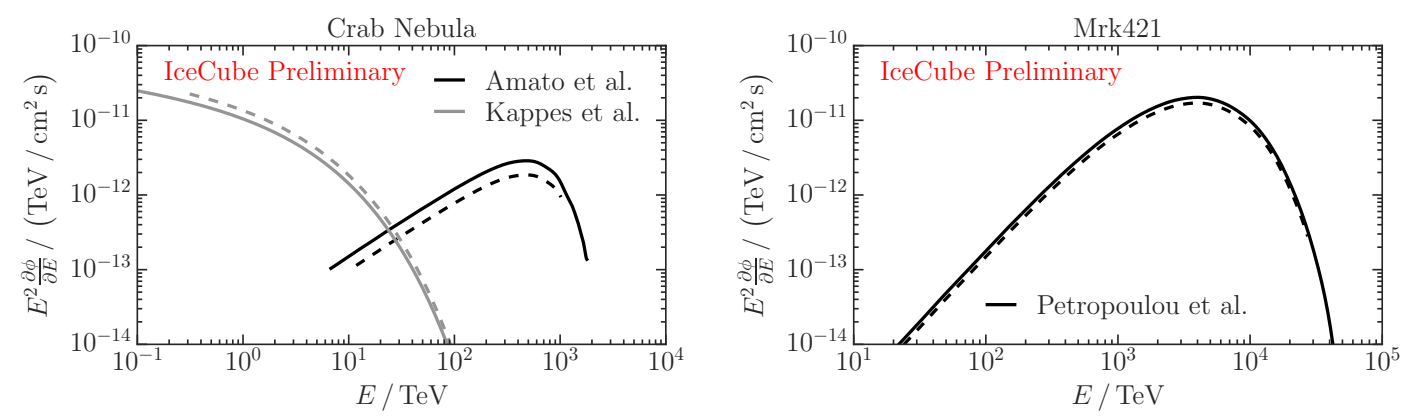

Figure 3: Model predictions of muon neutrino fluxes for the Crab nebula (PWN) [11, 12], and the BL Lac Mrk421 [13], both located in the northern hemisphere where IceCube is most sensitive. Solid lines indicate the model prediction, dashed lines the $90 \%$ upper limit with respect to the model.

In the analysis of the full sky and selected source lists, all results are consistent with pure background expectation. The most significant source PKS 1406-076 is connected to an over-fluctuation at a p-Value of 5.1\%. With the obtained sensitivity, IceCube can put stringent upper limits on model predictions for the Crab nebula and Mrk421. So far, IceCube's sensitivity is the best for neutrino point-like source emission in the TeV-PeV, and in the PeV-EeV in the northern-, southern sky, respectively. For the first time, the northern sky sensitivity reaches below $10^{-12} \mathrm{TeV} \mathrm{cm}^{-2} \mathrm{~s}^{-1}$ over most of the declination range of IceCube.

\section{References}

[1] IceCube Collaboration, A. Achterberg et al., Astropart.Phys. 26 (2006) 155-173, [astro-ph/0604450].

[2] IceCube Collaboration, M. Aartsen et al., Phys.Rev.Lett. 113 (2014) 101101, [arXiv: 1405.5303 ].

[3] IceCube Collaboration, M. Aartsen et al., Phys.Rev. D91 (2015), no. 2 022001, [arXiv:1410.1749].

[4] IceCube Collaboration, M. Aartsen et al., Evidence for astrophysical neutrinos from the northern sky with IceCube, submitted to PRL, 2015.

[5] IceCube Collaboration, L. Rädel and S. Schoenen, POS(ICRC2015)1079 these proceedings (2015).

[6] IceCube Collaboration, M. Aartsen et al., Astrophys.J. 779 (2013) 132, [arXiv:1307. 6669].

[7] IceCube Collaboration, M. Aartsen et al., arXiv:1406.6757.

[8] T. K. Gaisser, K. Jero, A. Karle, and J. van Santen, Phys.Rev. D90 (2014), no. 2023009 , [arXiv:1405.0525].

[9] IceCube Collaboration, R. Ström, PoS(ICRC2015)1053 these proceedings (2015).

[10] IceCube Collaboration, D. Altmann and A. Kappes, PoS(ICRC2015)1056 these proceedings (2015).

[11] A. Kappes et al., The Astrophysical Journal 656 (2007), no. 2870.

[12] E. Amato, D. Guetta, and P. Blasi, Astron.Astrophys. 402 (2003) 827-836, [astro-ph/ 0302121 ].

[13] M. Petropoulou et al., Mon.Not.Roy.Astron.Soc. 448 (2015), no. 4 3121-3131, [arXiv:1501.07407]. 